\title{
Macrobending loss properties of photonic crystal fibres with different air filling fractions
}

Sørensen, Thorkild; Broeng, Jes; Bjarklev, Anders Overgaard; Knudsen, Erik; Libori, Stig E. Barkou; Simonsen, Harald R.; Jensen, Jacob Riis

\section{Published in:}

Proceedings on 27th European Conference on Optical Communication

Link to article, DOI:

10.1109/ECOC.2001.989675

Publication date:

2001

Document Version

Publisher's PDF, also known as Version of record

Link back to DTU Orbit

Citation (APA):

Sørensen, T., Broeng, J., Bjarklev, A. O., Knudsen, E., Libori, S. E. B., Simonsen, H. R., \& Jensen, J. R. (2001). Macrobending loss properties of photonic crystal fibres with different air filling fractions. In Proceedings on 27th European Conference on Optical Communication (Vol. 3, pp. 380-381) https://doi.org/10.1109/ECOC.2001.989675

\section{General rights}

Copyright and moral rights for the publications made accessible in the public portal are retained by the authors and/or other copyright owners and it is a condition of accessing publications that users recognise and abide by the legal requirements associated with these rights.

- Users may download and print one copy of any publication from the public portal for the purpose of private study or research.

- You may not further distribute the material or use it for any profit-making activity or commercial gain

- You may freely distribute the URL identifying the publication in the public portal 


\title{
Macrobending loss properties of photonic crystal fibres with different air filling fractions
}

\author{
Thorkild Sørensen (1), Jes Broeng (1), Anders Bjarklev (1), Erik Knudsen (1), \\ Stig E. B. Libori (1), Harald R. Simonsen (2), and Jacob Riis Jensen (2)
}

(1) COM, Technical University of Denmark, DTU, Building 345v, DK-2800 Kgs. Lyngby, Denmark Web address: www.com.dtu.dk, Phone: +454525 3808, Fax: + 4545936581 (thors@com.dtu.dk)

(2) Crystal Fibre A/S, Blokken 84, DK-3460 Birkerød, Denmark

Web address: www.crystal-fibre.com, Phone: +454348 2800, Fax: +4543482801

\begin{abstract}
We present experimental and theoretical analysis of macrobending losses of photonic crystal fibres with various air filling fractions. A scalar, effective-index method provides a good description of the losses for fibres with limited air filling fractions, whereas the method overestimates the losses for fibres with larger air filling fractions.
\end{abstract}

\section{Introduction}

Photonic Crystal Fibres (PCFs) are optical fibres made from undoped silica $[1,2]$. Their waveguiding is provided by a pattern of air holes distributed in the cladding, and these holes run along the entire fibre length. The arrangement of air holes may result in two different types of guiding principles, namely guiding by modified total internal reflection (M-TIR) and the photonic bandgap (PBG) effect [2]. M-TIR is analogous to total internal reflection, known from standard fibres, whereas the PBG effect is only seen in PCFs, where its guiding properties appear as a result of a periodic, microstructured cladding, in which a certain wavelength range is denied propagation [2]. Today PCFs that operate by M-TIR has been most widely studied, because this principle requires lower accuracy in its manufacturing process.

One of the most important issues regarding practical development of PCFs concerns their macrobending loss properties. For a wide range of applications, coiled-up special fibres are used - and for PCFs also to find a way into special fibre components, research attention must, naturally, be paid to macrobending losses. Here, we present experimental verification of a theoretical model capable of analysis of macrobending losses in PCFs. We provide important insight into bending properties of PCFs, including the spectral windows that the fibres can be operated in, and the design parameters that determine these windows. The model is scalar and based on the weakly guiding approximation and we explore its validity for both low and high air filling fraction PCFs.

Theory and experiments

A bent PCF is schematically shown in fig. 1. The end facet shows a scanning electron micrograph of one of the fibres under test. The distance between the air holes is denoted the pitch, $\Lambda$, and the diameter of the air holes is denoted $d$. In order to deduct a theoretical tool for handling macrobending losses in PCFs, we apply first an analogy to step-index fibres, introducing an effective normalized frequency for PCFs, $V_{\mathrm{eff}}$, as presented initially by Birks et al. [3]:

$$
V_{e f f}=\frac{2 \pi \rho_{e q}}{\lambda} \sqrt{n_{c o}{ }^{2}-n_{c l, e f f}^{2}}
$$

Here, $\rho_{\mathrm{eq}}$ is the core radius of an equivalent step-index fibre having similar properties as the PCF at wavelength $\lambda . n_{c o}$ is the refractive index of the core, and $n_{\text {eff }}$ is the effective refractive index of the PCF cladding region. $n_{\text {cleetr }}$ has a strong wavelength dependency due to the air hole array $[2,3]$. At short wavelengths, the field is mainly confined to the silica, whereas at long wavelengths it has an effective index, determined by the air filling fraction of the cladding. This implies that the refractive-index difference decreases with wavelength, and that the field confinement factor of a guided mode will decrease as well, resulting in the unusual property of high PCF bending losses at short wavelengths. $n_{\text {cleteff }}$ has been calculated by an effective-index approach, using the amount of field present in the holes and in the silica [2,3], and the wavelength dependence of the refractive index of silica has been taken into account in the model. In its present form, the model is a scalar, semianalytic model and it approximates the hexagonal-like core shape of the PCF with a circular shape [3].

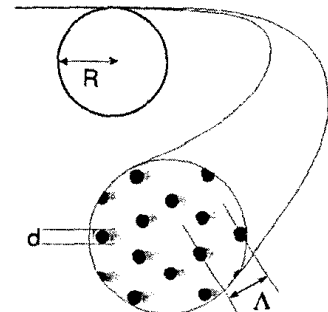

Fig. 1: Schematic of a bent PCF

For proper use, the model requires a specific core radius of an equivalent step-index fibre to be chosen. Empirically, an optimum equivalent core radius for a PCF with a design as shown in Fig. 1 has been found to be $\rho=0.62 \wedge[4]$. To proceed deducing a useful bending loss description, a formula for the power loss coefficient of standard stepindex fibres due to macro bending $[5,6]$ is applied:

$$
\alpha=\frac{\sqrt{\pi} \rho_{e q} A_{e}^{2} \exp \left(\frac{-4 \Delta_{e f f} W_{e f f}^{3} R}{3 \rho_{e q} V_{e f f}^{2}}\right)}{8 P W_{e f f} \sqrt{\frac{W_{e f f} R}{\rho_{e q}}+\frac{V_{e f f}^{2}}{2 \Delta_{e f f} W_{e f f}}}}
$$

where $\Delta_{\text {eff }}$ is the relative difference between the core and the effective cladding indices, $V_{e f l}$ is the effective normalized frequency, $\rho_{c u}$ is the equivalent core radius and $\mathrm{W}_{\text {eff }}$ is the normalized decay parameter of the cladding. $\mathrm{R}$ denotes the radius of curvature in the bend, $A_{c}$ is the amplitude coefficient of the electric field in the cladding, and, finally, $\mathrm{P}$ is the power carried by the fundamental mode. Utilizing this formula, we have calculated the macrobending loss from the coefficients of the Bessel function of the equivalent step-index fibre. The wavelength dependencies of $\Delta_{\text {eff }}$ and $V_{\text {eff }}$ correctly predict a loss 
mechanism for shorter wavelengths as found in [3]. As seen in figure 2, a short-wavelength loss edge appears for PCFs. This is in contrast to the standard fibre case, where only a long-wavelength loss edge is found. As seen from the figure, the two loss edges determine spectral windows in which PCFs can be operated. Fig. 2 further illustrates the bending radius dependency of the operational windows for a specific PCF with $d=2.4 \mu \mathrm{m}$ and $\Lambda=7.8 \mu \mathrm{m}$.

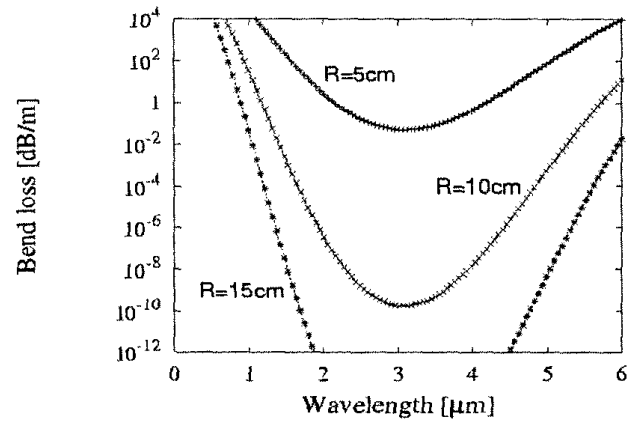

Fig. 2: Simulation of macrobending loss of the PCF in Fig. 1 for 3 different bending radii. The PCF exhibits both a bend loss edge at long wavelengths - as well as at short wavelength.

In order to verify the predictions of the semi-analytical, effective-index model, a PCF with $d=2.4 \mu \mathrm{m}$ and $\Lambda=7.8$ $\mu \mathrm{m}$ was fabricated. The silica-air fibre had $125 \mu \mathrm{m}$ outer diameter and polymer coating making it mechanically robust. The macrobending properties of the fibre were characterized experimentally, and the results are illustrated in Fig. 3. The figure further includes the prediction of the effective-index model, and as seen the model is capable of predicting accurately the spectral location of the (unusual) lower bending loss edge. The upper bending loss edge of the specific fibre is for all bending radii positioned at midinfrared wavelengths.

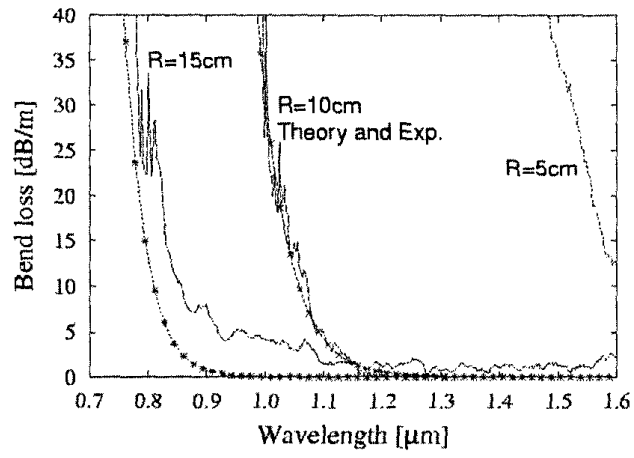

Fig. 3: Measured and simulated lower macrobending loss edge for PCF of Fig. 1. For operation around 1.5 $\mu \mathrm{m}$, the critical bending radius is around $6 \mathrm{~cm}$.

While Fig. 2 and 3 present an analysis of a specific PCF chosen particularly to investigate the unusual property of a short-wavelength loss edge and to illustrate the capability of an effective index model - we have also determined a number of general characteristics regarding macrobending properties of PCFs. Apart from the bending radius, the spectral width and positioned of the operational window depends strongly on hole size and pitch. Generally, large hole size results in broader windows, whereas the pitch roughly determines the centre position of the window (as a first approximation the minimum bend loss occurs at a wavelength around $\Lambda / 2$ ).
To explore the validity of the scalar model also for PCFs with higher air filling fractions, a large mode area $\mathrm{PCF}$ having $\Lambda=10 \mu \mathrm{m}$ and $\mathrm{d}=5.5 \mu \mathrm{m}$ was examined. The PCF was found to be single mode from visible to near-infrared wavelengths. The measured macrobending losses of this $\mathrm{PCF}$ are illustrated in Fig. 4. From the figure it is seen that the PCF is significantly more robust with respect to macrobending compared to the previously studied PCF. We attribute this to the increased air filling fraction. The increased air filling fraction, however, also makes this PCF more difficult to model using the scalar approach utilizing the weakly waveguiding approximation. Indeed, we find that the lower macrobending loss edge is less well predicted for this PCF. The simulated loss edge at a bending radius of $1.2 \mathrm{~cm}$ shows that the scalar model predicts a higher loss than what is actually found for the real PCF.

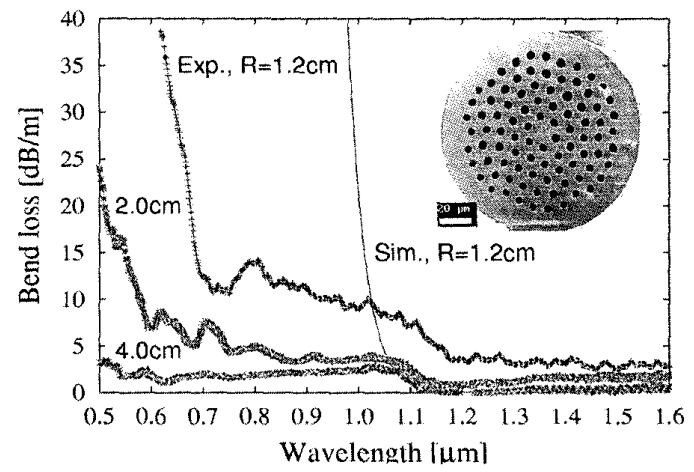

Fig 4: Measured and simulated lower macrobending loss edge for PCF shown in the inset. The PCF has a core diameter of $15 \mu \mathrm{m}$ and a relatively large air filling fraction. For operation at wavelengths above $1.2 \mu \mathrm{m}$, the PCF is single mode and in practice insensitive to macrobending.

\section{Summary}

An effective-index model has been applied to PCFs when subject to bending. Experimental bend loss characterizations have been performed and validated the possibility of qualitative prediction of macrobending losses in PCFs. In its present scalar form, the model provides accurate results for PCFs with relatively small air filling fractions, whereas it overestimates the macrobending losses for PCFs with larger air filling fractions. To further improve the model, the effective cladding index should be determined using a full-vectorial calculation method and the mode solutions for the equivalent step-index fibre should be found without use of weakly waveguiding approximations. Future results using such improvements for modeling of macrobending losses in PCFs with large air filling fractions will be presented.

\section{References}

11/ Knight et al., Opt. Lett., vol. 21, pp. 1547-1549 1996.

12/ Broeng et al., Optical Fiber Technology, vol. 5, pp. 305-330, 1999.

13/ Birks et al., Opt. Lett., vol. 22, pp. 961-963, 1997.

14/ Birks et al,, Optical Fiber Communication Conference, FG4 vol.4, pp.114-116, 1999.

15/ Sakai et al., Applied Optics, vol. 17, no. 10, pp. 1499$1506,1978$.

16) Knudsen et al., $14^{\text {th }}$ International Conference on Optical Fiber Sensors OFS2000, 11-13 October 2000, pp 904-907. 